\title{
IMMUNOGENETICS OF SELF-INCOMPATIBILITY IN BRASSICA OLERACEA L.*
}

\author{
M. E. NASRALLAH and D. H. WALLACE $\dagger$ \\ Department of Plant Breeding, Cornell University, Ithaca, New York
}

Received 8.xii.66

\section{INTRODUCTION}

IN many plant species the control of self-incompatibility is attributed to a single locus known as the $S$ locus. Most species thus far investigated have multiple alleles at this locus, designated as $S_{1}, S_{2} \ldots S_{n}$. Some of these $S$ alleles, designated as $S_{f}$ (East, I929), and some non-allelic genes (Tseng, 1938) condition self-fertility. The estimated number of $S$ alleles exceeds 200 in some species (Bateman, 1947). Hypotheses concerning the mechanism of control of the incompatibility reaction have been derived mainly from genetic analysis. The control is such that presence of identical alleles in pollen and pistil results in incompatibility. Thus, $S_{1}$ pollen fails on $S_{1}$ plants but presumably functions normally on $S_{1}, S_{2} \ldots S_{n}$. In the absence of dominance, which is commonly present in species with sporophytic control, but usually absent in species with gametophytic control (Pandey, 1960), an $S_{1} S_{2}$ plant cannot be fertilised by either $S_{1}$ or $S_{2}$ pollen. Selfincompatibility is in essence a mating control device which physiologically restricts inbreeding in both higher plants and fungi.

Very little is known of the biochemistry by which female organs recognise specific pollen genotypes. A priori, the extensive genetic variability at the $S$ locus should also be manifest as biochemical variability. Several methods for detecting such biochemical variability have been attempted but, so far, immunochemical techniques seem most promising. Lewis (1952) used serological techniques to identify incompatibility substances; he concluded that the different $S$ alleles produce specific and antigenically distinguishable substances in pollen of Oenothera organensis. Linskens ( 1960 ) extended Lewis's results and reported that the pollen and styles of Petunia have identical antigens as was earlier predicted by East (1929). Mäkinen and Lewis (1962) used immunodiffusion techniques and verified the earlier work.

This paper reports the use of immunodiffusion to detect antigens, whose presence is attributable to alleles at the $S$ locus, in the stigmas of cabbage, Brassica oleracea var. capitata. Data supporting involvement of these antigens in the incompatibility reaction are presented. Cultivated cabbage offers the following advantages for studies of the biochemistry of incompatibility: (a) Immature cabbage flowers (buds),

* Department of Plant Breeding Paper No. 526 .

$\dagger$ Assistant Professor of Plant Breeding and Associate Professor of Plant Breeding and Vegetable Crops, Cornell University, Ithaca, New York. 
unlike mature flowers, are self-compatible. Thus, homozygous $S$ allele genotypes can be obtained by bud pollination. (b) At least 20 $S$ alleles have been studied genetically. Many of these are available as homozygous inbreds, as are numerous other inbreds from plant breeding programmes. (c) Flowers are produced abundantly; several hundred can be collected from a single plant. (d) The pollen tubes are inhibited on the stigmatic surface and therefore the site and tissues involved in the incompatibility reaction are both specific and more amenable to biochemical analysis than is true with many other species. According to Christ (I959), the incompatibility effect on pollen grains is noticeable in less than 20 minutes in Cardamine pratensis, which belongs to the family Crucifere as does Brassica oleracea.

\section{MATERIALS AND METHODS:}

Inbreds: Three self-incompatible inbreds $S_{1} S_{1}, S_{2} S_{2}$ and $S_{3} S_{3}$ were used. Inbreds $S_{1} S_{1}$ and $S_{2} S_{2}$ were derived from a single plant and have been maintained by selfing in the bud stage. Inbred $S_{3} S_{3}$ is unrelated; it has been inbred for nine generations. In 1962 a naturally occurring self-fertile mutant was isolated from the $S_{2} S_{2}$ inbred. This will be referred to as $S_{2}^{\prime} S_{2}^{\prime}$. The self-fertility was found to be controlled by a single dominant gene (unpublished data). Other inbreds that provided test antigens or were used in crosses with the aforementioned inbreds will be described in the text.

Antisera. Antisera were produced in rabbits against stigmatic homogenates of $S_{1} S_{1}, S_{2} S_{2}$ and $S_{3} S_{3}$ and will be designated as $A H S_{1}, A H S_{2}$ and $A H S_{3}$ respectively. The homogenates were prepared from stigmas that were extracted for five minutes in a mortar and pestle using buffer-saline ( $O_{1}$ M phosphate buffer and $0_{1} \cdot{ }_{5} \mathrm{M} \mathrm{NaCl}$ ) and were then centrifuged for 20 minutes at $10,000 \mathrm{~g}$. and $\mathrm{o}^{\circ} \mathrm{C}$.

$A H S_{1}$ was produced by a combination of nine intravenous and two subcutaneous injections. The latter injections were administered at several sites in the nuchal region and consisted of a I:I mixture of stigmatic homogenate and Difco's complete Freund's adjuvant. Extracts from a total of goo stigmas, in $5 \mathrm{ml}$. of solution, were injected into one rabbit over a four-week period and the serum was harvested a week after the last injection.

$A H S_{2}$ was produced by repeated intravenous injections. Twelve injections were administered in four weeks and extracts from a total of 1500 stigmas were used per rabbit. $A H S_{3}$ was similarly obtained except that only half as many stigmas were utilised.

Double diffusion. The agar diffusion medium was I per cent. Difco Special Noble agar containing $0_{5} 5$ per cent. sodium azide ( $\mathrm{pH}$ of this solution was approximately 7 ). Measured portions of melted agar were poured to a depth of $2.5 \mathrm{~mm}$. in flatbottomed petri dishes or on $3 \mathrm{~cm} . \times 10 \mathrm{~cm}$. microscope slides. After the agar had set, wells were cut with precisely spaced gel cutters. The agar was then lifted by suction from the wells and a small amount of melted agar was placed in each well to seal the bottom and prevent seepage beneath the agar layer. The diameter of the antiserum well was $0.8 \mathrm{~cm}$. and that of the antigen wells $0.4 \mathrm{~cm}$. The antigen wells were positioned at a distance of $0.4 \mathrm{~cm}$. from the serum well. Undilited sera were used in all tests. After charging the centre well with antiserum $(0.2 \mathrm{ml}$.) and the outer wells with antigens $\left(0^{\circ} 015 \mathrm{ml}\right.$.), the plates were kept at $5^{\circ} \mathrm{C}$. until precipitation bands became visible.

Immunoelectrophoresis (IEP). IEP was performed on glass plates according to Grabar (1959). The antigens were subjected to electrophoresis in a constant current

$¥$ Inbreds $S_{1} S_{1}, S_{2} S_{2}, S_{2}^{\prime} S_{2}^{\circ}$ and $S_{f} S_{f}$ were referred to in Nasrallah and Wallace (ig67) as inbreds $A, C, D$ and $E$ respectively. 
for $\mathrm{I} \frac{1}{2}$ hours at 6 volts $/ \mathrm{cm}$. Barbital buffer $(\mathrm{pH} \mathrm{8 \cdot 2)}$ was used for extracting the antigens, for preparation of the agar gel, and as the electrolyte solution in electrophoresis.

Absorption. Double diffusion tests were also conducted with absorbed antiserum. Absorption was carried out as follows: homogenised stigmas (3o stigmas in $o^{\prime} \mathrm{I} \mathrm{ml}$. of buffer-saline) were centrifuged for I 5 minutes at $2000 \mathrm{~g}$ and one volume of the homogenate was mixed with three volumes of antiserum. This mixture was incubated at $25^{\circ} \mathrm{C}$. for one hour and then stored overnight in the refrigerator. The mixture was again centrifuged at $2000 \mathrm{~g}$ for $\mathrm{I} 5$ minutes and the supernatant was used to test for unabsorbed antibody components.

Test antigens. These were obtained from freshly collected stigmas, and will be referred to as $H S_{1}, H S_{2}$ and $H S_{3}$, indicating homogenates of $S_{1} S_{1}, S_{2} S_{2}$ and $S_{3} S_{3}$ stigmas. Twenty-five stigmas, homogenised in $\mathrm{O}^{\prime} \mathrm{I} \mathrm{ml}$. buffer saline, were found to give a satisfactory concentration for testing with $A H S_{1}$ and $A H S_{3}$, while half as many stigmas were adequate with $A H S_{2}$.

\section{RESULTS}

Figure I is a photograph of an immunodiffusion plate where $A H S_{2}$ has reacted with $H S_{2}, H S_{1}$ and $H S_{3}$. A reaction of nonidentity represented by the middle band is evident opposite $H S_{2}$, indicating that $H S_{2}$ is antigenically distinguishable from $H S_{1}$ and $H S_{3}$. This was later verified by absorbing $A H S_{2}$ with $H S_{1}$ (heterologous absorption) and allowing the absorbed $A H S_{2}$ to react with $H S_{1}, H S_{2}$ and $H S_{3}$. Figure 2 shows results of such a test. Only one band was formed; since it was opposite the $H S_{2}$ wells it will be referred to as the $S_{2}$ band. Absorption of $A H S_{2}$ with $H S_{3}$ gave results similar to those of fig. 2 while homologous absorption with $\mathrm{HS}_{2}$ eliminated all bands.

A reaction of nonidentity was also observed in tests with $A H S_{1}$ when $H S_{1}$ was adjacent to either $H S_{2}$ or $H S_{3}$. This indicated that $H S_{1}$ contained an antigen or antigens unique to the $S_{1} S_{1}$ genotype as was verified by the absorption of $A H S_{1}$ with $H S_{2}$ (fig. 3). This figure shows the reaction of absorbed $A H S_{1}$ with $H S_{1}, H S_{2}$ and $H S_{3}$. One band was detected opposite $H S_{1}$ and none opposite $H S_{2}$ or $H S_{3}$. Absorption of $A H S_{1}$ with $H S_{3}$ gave results similar to those of fig. 3, while absorption with $H S_{1}$ eliminated all bands. Immunoelectrophoretic tests with unabsorbed $A H S_{1}$ were in agreement with the above conclusions as shown in fig. 4, where $H S_{2}$ (well 2) and $H S_{1}$ (well I) were first subjected to electrophoresis and then allowed to react with $A H S_{1}$. Eight bands were detected with $H S_{2}$ as opposed to nine with $H S_{1}$. The concentration of IEP test antigens was I oo stigmas extracted in $O^{\cdot I} \mathrm{ml}$. of buffer. The immunoelectrophoretic pattern obtained with $H S_{3}$ was similar to that of $H S_{2}$, thus indicating that $H S_{1}$ has at least one unique antigen detectable with $A H S_{1}$.

Tests with $A H S_{3}$ gave results similar to those of $A H S_{1}$ and $A H S_{2}$ in the sense that $H S_{3}$ was found to contain a unique antigen that could not be detected in $H S_{1}$ or $H S_{2}$. Figure 5 shows the reaction of $S_{3}$ and $S_{2}$ stigmas with $A H S_{3}$. The stigmas were lifted from the style, by exerting pressure at the base of the stigma, and used as test antigens since it was known that certain stigmatic antigens are freely diffusible 
(Nasrallah and Wallace, r 967 ). One additional antigenic component is detected opposite $S_{3}$ stigmas but not opposite $S_{2}$. Stigmatic homogenates used as test antigens against absorbed and unabsorbed $A H S_{3}$ support this conclusion although $A H S_{3}$ had a low titre which resulted in weak precipitation bands.

Detection of antigens in the $F_{1}$ hybrids. Homogenates of stigmas obtained from the three possible hybrids, $S_{1} S_{2}, S_{1} S_{3}$ and $S_{2} S_{3}$, were allowed to react with $A H S_{1}, A H S_{2}$ and $A H S_{3}$. Such tests revealed that hybrid stigmas contain the two parental $S$ antigens (table I). In correlation with the presence of stigmatic $S$ antigens, the $F_{1}$ genotypes rejected pollen from their respective parents while accepting pollen from the non-parental inbred.

TABLE :

Reaction of hybrid stigmas with antisera prepared against parental genotypes

\begin{tabular}{|c|c|c|c|c|}
\hline & & \multicolumn{3}{|c|}{ Genotype of hybrid Stigma } \\
& \multirow{2}{*}{ Antiserum } & Absorbing antigen & & \\
& & $H S_{1} S_{2}$ & $H S_{1} S_{3}$ & $H S_{2} S_{3}$ \\
\hline & & + & + & - \\
\hline$H S_{1}$ & $\begin{array}{c}H S_{2} \text { or } H S_{3} \\
H S_{1} \text { or } H S_{3} \\
H S_{2}\end{array}$ & + & + & + \\
\hline$H S_{3}$ or $H S_{2}$ & - & + & + \\
\hline
\end{tabular}

Figure 6 shows detection of the $S_{2}$ antigen opposite $H S_{2}, H S_{2} S_{2}^{\prime}$ and $H S_{2} S_{3}$ when these were allowed to react with absorbed $A H S_{2}\left(H S_{3}\right.$ was the absorbing antigen). The bands formed a symmetrical hexagon indicating that equal concentrations of the $S$ antigen were present in $S_{2} S_{2}, S_{2} S_{2}^{\prime}$ and $S_{2} S_{3}$ stigmas. Similar results were obtained with $S_{1} S_{2}$.

Tissue specificity. As indicated above, heterologous absorption of each of $A H S_{1}, A H S_{2}$ and $A H S_{3}$ led to the detection in $H S_{1}, H S_{2}$ and $H S_{3}$ of unique and serologically distinguishable antigens designated as the $S$ antigens. The question was then raised as to whether these antigens are present in other plant parts. Test antigens were prepared from styles, ovary, anthers including pollen, embryos and leaves, and were allowed to react against the aforementioned sera. No $S$ antigens were detected in any of these tissues. $A H S_{1}, A H S_{2}$ and $A H S_{3}$ were also absorbed with anther-pollen homogenates of $S_{1}, S_{2}$ and $S_{3}$ respectively, but the absorbed sera reacted with the $S$ antigens and gave precipitation bands with the homologous stigmatic homogenate. These results indicate that neither anthers nor pollen nor any tissue other than stigmas contain detectable $S$ antigens.

Properties of the $\mathrm{S}_{2}$ antigen. For more detailed studies of the chemical properties of $S$ antigens, $S_{2}$ was used, because sufficient plant material was available and because $A H S_{2}$ had high titre which made the assay more simple. $H S_{2}$ was easily denatured by boiling or even by heating at $70^{\circ} \mathrm{C}$. for ten minutes. The $S_{2}$ antigen was also precipitated by 
saturated $\left(\mathrm{NH}_{4}\right)_{2} \mathrm{SO}_{4}$ solution, details of this procedure being found in Nasrallah (1965). Another property of both $S_{1}$ and $S_{2}$ antigens was their migration to the cathode at $\mathrm{pH} 8 \cdot 2$ (fig. 4). This suggests that these molecules might be highly basic, although the migration to the cathode could be due in part to electro-osmosis. This property is being investigated further. One other property of the $S$ antigens is their relative stability. $H S_{1}, H S_{2}$ and $H S_{3}$ solutions have been stored for several weeks at $-\mathrm{I}^{\circ} \mathrm{C}$. and alternate freezing and thawing have had no observable effect on the ability of these antigens to react with the respective antisera.

Antigens in the self-fertile mutant $\mathrm{S}_{2}^{\prime}$. The self-fertility of $S_{2}^{\prime}$ was found to be controlled by a single nonallelic gene. Unilateral cross-incompatibility resulted from this mutation, as reflected by incompatibility in the cross $S_{2}$ ㅇ $\times S_{2}^{\prime}{ }^{\star}$ but compatibility in the reciprocal cross. The cross-pollination data indicated that the pollen specificity was unchanged and the unilateral behaviour was attributed to a change in the stigmatic specificity. However, it was shown that $H S_{2}^{\prime}$ did react with the $S_{2}$ antibodies, but not to the same degree as $H S_{2}$. This conclusion is based on the following tests: (a) Homogenates, containing the same number of stigmas of $S_{2}$ and $S_{2}^{\prime}$, and used to absorb $A H S_{2}$, led to complete antibody absorption with the former antigen but only partial absorption with the latter. (b) Double diffusion tests showed that the $S_{2}$ band opposite the $H S_{2}^{\prime}$ well was displaced towards the antigen source as compared to the band formed opposite $H S_{2}$, which formed closer to the serum well. Moreover, the $S$ band became visible in less than 24 hours when $H_{2}$ was the test antigen as compared to 36 hours for $H S_{2}^{\prime}$. Both observations are based on adjacent well comparisons. (c) Serial dilutions of $H S_{2}$ and $H S_{2}^{\prime}$ indicated the presence at high dilution of an $S$ band opposite $H S_{2}$ when no $S$ band could be detected opposite $H S_{2}^{\prime}$. Figure 7 shows results of such a dilution experiment whereby $35^{\circ}$ stigmas of each of $S_{2}$ and $S_{2}^{\prime}$ were freeze-dried, weighed and extracted in appropriate volumes of buffer. $S_{2}$ and $S_{2}^{\prime}$ stigmas weighed 0.01895 and 0.02075 gram respectively; the former were extracted in $0.3790 \mathrm{ml}$. and the latter in $0.4150 \mathrm{ml}$. The supernatant of each homogenate was diluted ten times and then used as test antigen with absorbed $A H S_{2} \quad\left(H S_{1}\right.$ was the absorbing antigen). Figure 7 shows that a band appears opposite $H S_{2}$ (left side of the figure) but not opposite $H S_{2}^{\prime}$ (right), although the $S_{2}$ band bends towards the $S_{2}^{\prime}$ antigen indicating that minute quantities of $S_{2}$ antigen are present in $H S_{2}^{\prime}$ wells. The same diluted homogenates were tested according to the quantitative assay methods of Hayward and Augustin (r957). For these tests $A H S_{2}$ was incorporated into the gel by mixing one part serum with one part of 2 per cent. agar solution at $54^{\circ} \mathrm{C}$. This mixture was poured into a petri dish and various quantities of $H S_{2}$ and $H S_{2}^{\prime}$ ( $1,2,5$ and 7 microliters) were placed in wells as shown in fig. 8 . The diameters of the precipitation halos are larger for $H S_{2}$ than for $H S_{2}^{\prime}$, indicating a greater concentration or reactivity for $H S_{2}$. 
Action of the $\mathrm{S}$ antigens. The detection of antigenic differences led to investigation of the inheritance of these differences and attempts were made to correlate the presence or absence of a given $S$ antigen in $F_{2}$ plants with incompatibility behaviour. The data of table 2 indicate such correlations. $\mathrm{F}_{2}$ progenies were obtained by selfing each of the following hybrids: $\left(S_{1} \times S_{2}^{\prime}\right),\left(S_{1}^{*} \times S_{2}^{\prime}\right),\left(S_{1} \times S_{2}\right),\left(S_{2} \times S_{f}\right)$,

TABLE 2

Correlation of the incompatibility genotype of $\mathrm{F}_{2}$ plants as determined by serology and by crosspollination

\begin{tabular}{|c|c|c|c|c|c|}
\hline \multirow{2}{*}{ Cross $\left(F_{1}\right)$} & \multirow{2}{*}{$\begin{array}{l}\text { Number of } \\
F_{2} \text { plants }\end{array}$} & \multicolumn{2}{|c|}{ Reaction with } & \multirow{2}{*}{$\begin{array}{l}\text { Genotype } \\
\text { determined by } \\
\text { serology }\end{array}$} & \multirow{2}{*}{$\begin{array}{c}\text { Genotype } \\
\text { determined by } \\
\text { pollination }\end{array}$} \\
\hline & & $A H S_{1}$ & $A H S_{2}$ & & \\
\hline$S_{1} \times S_{2}^{\prime}$ & $\begin{array}{l}1 \\
3\end{array}$ & $\bar{t}$ & + & $\begin{array}{l}S_{2} S_{2} \\
S_{1} S_{2}\end{array}$ & $\begin{array}{l}S_{2} S_{2} \\
S_{1} S_{2}\end{array}$ \\
\hline$S_{1}^{*} \times S_{2}^{\prime}$ & I & - & + & $S_{2} S_{2}$ & $S_{2} S_{2}$ \\
\hline$S_{1} \times S_{2}$ & $\begin{array}{r}10 \\
13 \\
6\end{array}$ & $\stackrel{+}{+}$ & $\begin{array}{l}\bar{t} \\
+\end{array}$ & $\begin{array}{l}S_{1} S_{1} \\
S_{1} S_{2} \\
S_{2} S_{2}\end{array}$ & $\begin{array}{l}S_{1} S_{1} \\
S_{1} S_{2} \\
S_{2} S_{2}\end{array}$ \\
\hline$S_{2} \times S_{f}$ & $\begin{array}{l}1 \\
2\end{array}$ & $\begin{array}{l}\cdots \\
\cdots\end{array}$ & $\bar{t}$ & $\begin{array}{l}S_{f} S_{f} \\
S_{2} S_{?}\end{array}$ & $\begin{array}{c}S_{y} S_{f} \\
\cdots\end{array}$ \\
\hline$S_{2} \times S_{3}$ & $\begin{array}{r}1 \\
14\end{array}$ & $\begin{array}{l}\ldots \\
\ldots\end{array}$ & $\bar{t}$ & $\begin{array}{l}S_{3} S_{3} \\
S_{2} S_{?}^{* *}\end{array}$ & $\begin{array}{c}S_{3} S_{3} \\
\ldots\end{array}$ \\
\hline$S_{1} \times S_{3}$ & $\begin{array}{l}1 \\
5\end{array}$ & $\bar{t}$ & $\ldots$ & $\begin{array}{l}S_{3} S_{3} \\
S_{1} S_{?}^{* *}\end{array}$ & $\begin{array}{c}S_{3} S_{3} \\
\ldots\end{array}$ \\
\hline
\end{tabular}

** These plants were not tested with $A H S_{3}$.

$\left(S_{2} \times S_{3}\right)$ and $\left(S_{1} \times S_{3}\right) . \quad S_{1}^{*}$ and $S_{1}$ are from two related but crossincompatible and homozygous sublines both of whose stigmatic homogenates react identically with $A H S_{1}$. They differ in modifier genes conditioning a small difference in the mean number of seeds obtained from self-pollination (unpublished data). The individual $F_{2}$ plants were assayed for $S_{1}$ and $S_{2}$ antigens by testing stigmatic homogenates with absorbed $A H S_{1}$ and $A H S_{2}$ as in previous tests. Cross-compatibilities with the parents were then determined with reciprocal crosses to both parents. In the three $S_{1} \times S_{2}$ progenies, $\mathrm{F}_{2}$ plants having $S_{2}$ antigen but no $S_{1}$ were found to be reciprocally cross-incompatible with the $S_{2} S_{2}$ parent and reciprocally compatible with the $S_{1} S_{1}$ parent. Plants having $S_{1}$ antigen but no $S_{2}$ were reciprocally compatible with $S_{2} S_{2}$ and reciprocally incompatible with $S_{1} S_{1}$. Plants with both $S_{1}$ and $S_{2}$ stigmatic antigens exhibited cross-compatibilities with the parents that were identical to those of heterozygotes derived directly from hybridization. In such $S_{1} S_{2}$ heterozygote, $S_{2}$ was dominant over 
$S_{1}$ in the pollen but individual action (co-dominance) was exhibited in the stigma (Nasrallah, I965). The pollen phenotypes of $S_{1} S_{2}$ was therefore $S_{2}$, irrespective of the pollen genotype. The reciprocal cross-compatibilities of $F_{2}$ plants exhibiting both $S_{1}$ and $S_{2}$ antigens with the two parents were in complete agreement with this interpretation.

Similar reasoning gave predictions of $\mathrm{F}_{2}$ genotypes in the $\left(S_{2} \times S_{f}\right)$, $\left(S_{2} \times S_{3}\right)$ and $\left(S_{1} \times S_{3}\right)$ progenies. One plant from $S_{2} \times S_{f}$ had no $S_{2}$ antigen and was therefore classified as $S_{f} S_{f}$. This plant was both selffertile and reciprocally compatible with the $S_{2} S_{2}$ parent. The two plants exhibiting $S_{2}$ antigen were reciprocally cross-incompatible with $S_{2} S_{2}$ as would be expected of either $S_{2} S_{2}$ or $S_{2} S_{f}$ plants since $S_{2}$ is dominant in the pollen (Nasrallah, I965). From the $S_{2} \times S_{3}$ progeny one plant had no $S_{2}$ antigen and was classified as $S_{3} S_{3}$. This plant was reciprocally cross-compatible with the $S_{2} S_{2}$ parent and reciprocally was cross-incompatible with $S_{3} S_{3}$. The other I4 plants were crossincompatible when pollinated with $S_{2} S_{2}$ pollen. The genotypes of the $\left(S_{1} \times S_{3}\right)$ progeny were identified in a similar manner to those of $\left(S_{2} \times S_{3}\right)$.

\section{DISCUSSION}

This report deals with the detection of biochemical variability in stigmas of different incompatibility genotypes of Brassica oleracea. The self-incompatibility reaction is sporophytically controlled in the species (Haruta, 1962); nevertheless, the results are in agreement with the conclusions of Lewis (I952) and Linskens ( 1960 ), who worked with gametophytic systems. The genotypes $S_{1} S_{1}, S_{2} S_{2}$ and $S_{3} S_{3}$ were each found to be antigenically distinguishable by immunodiffusion tests utilising antisera produced in rabbits against stigmatic homogenates of each genotype. High titred rabbit antisera have been repeatedly secured against some genotypes, mainly $S_{2} S_{2}$. This step is essential for the successful application of immunochemical methods in as much as it increases the sensitivity of the reliability of these procedures. The sensitivity attained can best be illustrated by the fact that a single stigma (intact or homogenised) is sufficient for use as a test antigen and can thus be identified as of a specific genotype. The three $S$ alleles studied in detail are but a small sample of the existing $S$ alleles. It remains to be determined whether all $S$ alleles elicit specific antibody response. So far, no cross-reactions have been observed with the $S_{2}$ antibodies although more than ${ }_{5} 5$ different $S$ allele genotypes have been tested.

That the observed antigenic differences are genetically determined can be deduced from the expression of both parental $S$ antigens in $\mathrm{F}_{1}$ hybrids and their segregation in the $F_{2}$ progenies. Studies are in progress to determine whether these antigenic differences represent a series of allelic alternatives. One feature of the $S$ antigens is that they appear to be present in the hybrids at levels equivalent to those found 
in the parents, as judged from the position of the precipitation bands. Further evidence on the heritable nature of the $S$ antigens is deduced from the effect of the single gene mutant referred to as $S_{2}^{\prime} S_{2}^{\prime}$. The presence of a suppressor gene in homozygous state is associated with low levels or low reactivity of the $S_{2}$ antigens. Or alternatively, the $S_{2}^{\prime}$ gene could produce a cross-reactive protein that is immunologically indistinguishable from $S_{2}$ but reacts with less avidity with $A H S_{2}$. However, reduction in the detected level of the $S_{2}$ antigen in $S_{2}^{\prime} S_{2}^{\prime}$ does not necessarily lead to self-fertility in this inbred since the $F_{1}$ hybrid, $S_{2} S_{2}^{\prime}$, is self-fertile and, yet, high levels of $S_{2}$ antigen comparable to $S_{2} S_{2}$ are detected.

The specific reaction controlled by the $S$ antigen is not known at present although several hypotheses have been advanced (Lewis, I963; Linskens, 1963). Nevertheless, evidence has been presented which thoroughly implicates involvement of the $S$ antigens in the incompatibility reaction. It has already been shown that the appearance of a high level of $S_{2}$ antigen is correlated with a change in developing buds from self-compatibility to self-incompatibility (Nasrallah and Wallace, I967). The correlation of $S_{1}$ and $S_{2}$ antigens in $\mathrm{F}_{2}$ plants with cross-incompatibility behaviour provides additional and convincing support. Moreover, low levels of $S_{2}$ antigen in the self-fertile mutant $S_{2}^{\prime} S_{2}^{\prime}$ and in self-compatible immature buds of $S_{2} S_{2}$ are not without retarding effect on the rate of growth of the $S_{2}$ and $S_{2}^{\prime}$ pollen tubes, as compared with pollen of other " $S$ " allele genotypes (unpublished data).

Another feature of the $S$ antigens which may be of physiological significance is the rapid exocellular diffusion or secretion of these antigens. Mäkinen and Lewis ( 1962 ) reported that the pollen antigens of Oenothera are also diffusible and postulated that these antigens function at the surface of the pollen tubes. In cabbage, where incompatible pollen inhibition takes place at the stigmatic surface, it is conceivable that the $S$ antigens are secreted exocellularly and come in contact with or even penetrate the non-germinated pollen grains. The fact that the $S$ antigens could not be detected in the pollen of cabbage raises interesting questions concerning the nature of the pollen specificity. Most hypotheses on incompatibility assume identity of pollen and stigmatic antigens. This cannot be reconciled with our data unless it is assumed that the pollen antigens of cabbage, unlike Oenothera and Petunia, are associated with membranes and cannot be extracted by aqueous methods.

Many questions remain to be answered. Are the $S$ antigens polypeptides and does each $S$ allele produce a unique antigenic specificity by changing the amino acid composition of this polypeptide? Are these antigens enzymes, inhibitors, or substances that control gene activity? Do the $S$ antigens diffuse into the pollen grain and, if so, where are they localised? And finally, what is the nature of the pollen specificity? 

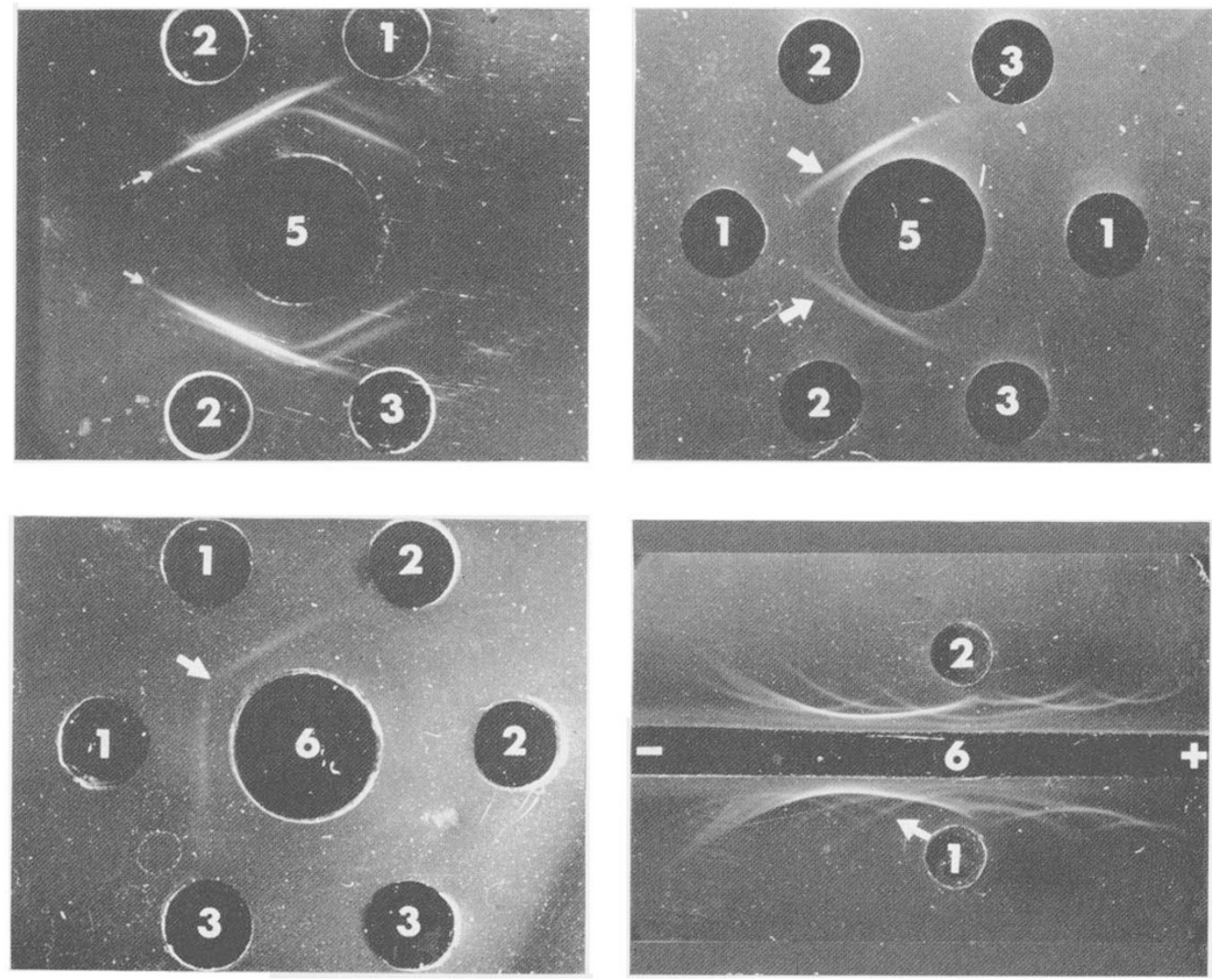

FIG. I.-Immunodiffusion in agar. Antigens $H S_{1}, H S_{2}$ and $H S_{3}$ (wells I, 2 and 3) were allowed to react with antiserum $A H S_{2}$ (well 5). The band indicated by the arrows is present only opposite $H S_{2}$.

Fig. 2.- Absorbed $A H S_{2}$ was placed in the centre well (5). Wells 1, 2, and 3 contained $H S_{1}, H S_{2}$ and $H S_{3}$. Absorption of $A H S_{2}$ with $H S_{1}$ eliminated all common antigens. the band opposite $H S_{2}$ is specific to the $S_{2}$ genotype.

Fig. 3.--Well 6 contained absorbed $A H S_{1}$ ( $H S_{2}$ was the absorbing antigen). Wells I, 2 and 3 contained $H S_{1}, H S_{2}$ and $H S_{3}$. The band indicated by the arrow is specific to the $S_{1}$ genotype.

FIG. 4.- Immunoelectrophoresis in agar at $\mathrm{pH} 8 \cdot 2$. $H S_{1}$ was placed in well I, $H S_{2}$ in well 2, and $A H S_{1}$ in the trough (6). The band indicated by the arrow is present only opposite $H S_{1}$. All other bands are common to $H S_{1}$ and $H S_{2}$. 

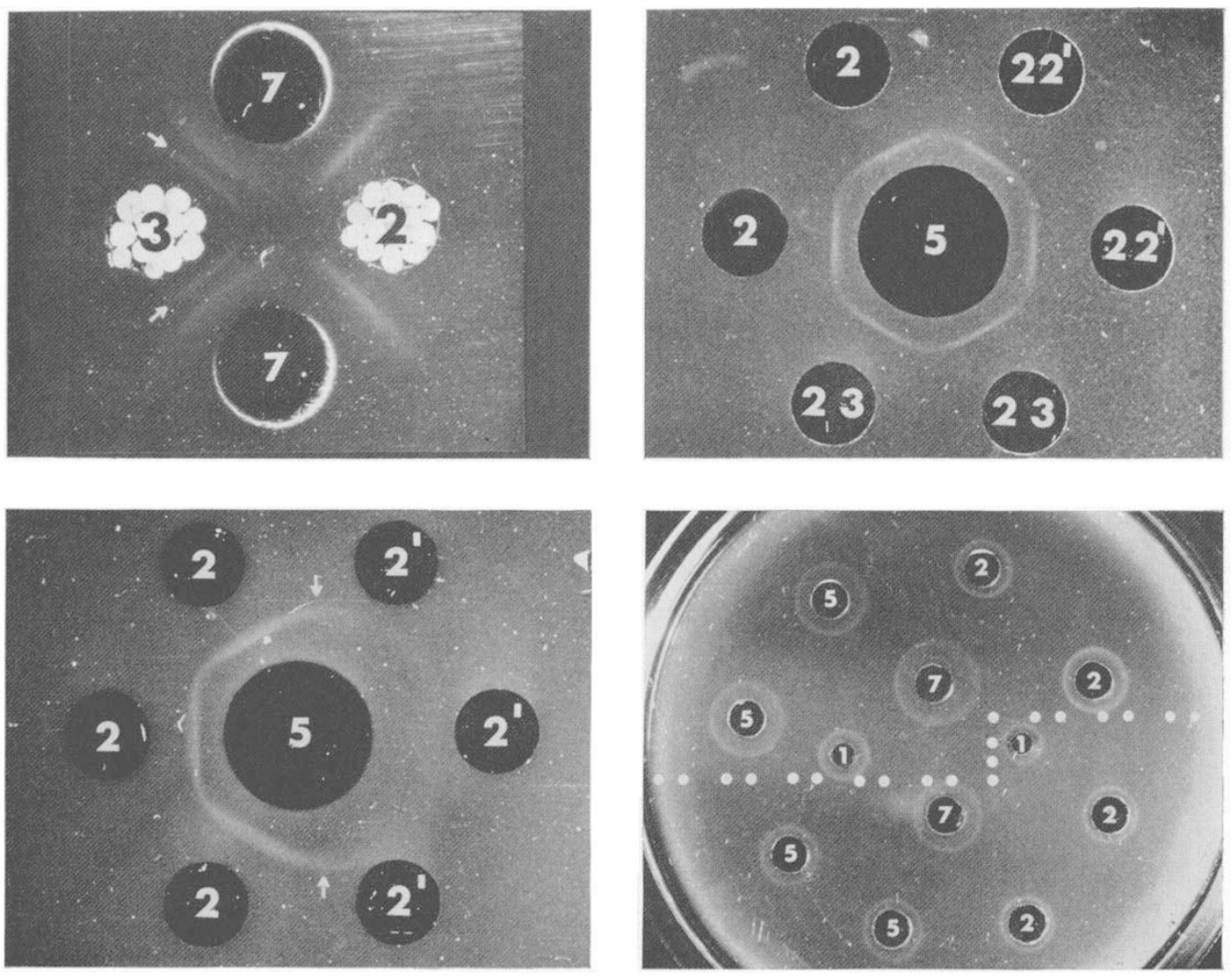

FIG. 5.- Intact stigmas used as test antigens. Well 7 contained $A H S_{3}$, wells 2 and 3 contained stigmas of $H S_{2}$ and $H S_{3}$. A genotype specific band (indicated by the arrows) appears opposite $H S_{3}$.

Fig. 6.- Absorbed $A H S_{2}\left(H S_{3}\right.$ was the absorbing antigen) was placed in the centre well (5). Wells 2, 22', and 23 contained $H S_{2}, H S_{2} S_{2}^{\prime}$ and $H S_{2} S_{3}$. The genotype specific band of $S_{2}$ is equidistant from homozygous (2) and heterozygous (22' and 23) wells, indicating equal concentration of the antigen.

Fig. 7.-Absorbed $A H S_{2}$ ( $H S_{1}$ was the absorbing antigen) was placed in the centre well (5). Wells numbered 2 and $2^{\prime}$ contained $H S_{2}$ and $H S_{2}^{\prime}$ of equivalent dilutions. One band appears opposite $H S_{2}$. The arrows point to the curvature of the band towards $H S_{2}^{\prime}$ indicating that small amounts of antigen are present in $H S_{2}^{\prime}$.

Fig. 8.-Unabsorbed $A H S_{2}$ was mixed with the agar. The homogenates of $H S_{2}$ and $H S_{2}^{\prime}$ used in fig. 7 are also compared here $\left(H S_{2}\right.$ above the dotted line and $H S_{2}^{\prime}$ below). The number of microliters $(1,2,5$ and 7 ) placed in each well is indicated. The halos of precipitation are larger for $H S_{2}$ at each corresponding antigen quantity, indicating higher antigen concentration or higher reactivity for $H S_{2}$ as compared with $H S_{2}^{\prime}$. 


\section{SUMMARY}

I. Immunodiffusion techniques were used to assay the antigens present in water-soluble homogenates of stigmas of different incompatibility genotypes of cabbage.

2. Antisera prepared in rabbits against stigmatic homogenates of three homozygous $\left(S_{1} S_{1}, S_{2} S_{2}\right.$ and $\left.S_{3} S_{3}\right)$, self-incompatible inbreds indicate that stigmas of each genotype have a specific and unique antigen whose presence is attributable to the specific $S$ allele. The genotype specific antigens of the stigma could not be detected in the pollen or in other tissues of the same plant.

3. Stigmas of the hybrids $S_{1} S_{2}, S_{1} S_{3}$ and $S_{2} S_{3}$ contained the two parental antigens and, in correlation with the presence of these antigens, they were incompatible with pollen from their respective parents.

4. For individual $\mathbf{F}_{2}$ plants, the genotype determined by the presence of either or both parental antigens was identical with that determined by reciprocal cross-pollination with the parents.

Acknowledgments.-The work was supported by a grant awarded by the National Science Foundation (Grant GB 4319). The authors acknowledge the assistance of Dr N. L. Norcross of the Veterinary College at Cornell.

\section{REFERENCES}

Bateman, A. J. 1947. Number of $\mathrm{S}$ alleles in a population. Nature, 160, 337.

CHRIST, B. I959. Entwicklungs geschichtliche und physiologische Untersuchungen uber die Selbststerilität von Cardamine pratensis L. Zeitschr. f. Bot., 47, 88- I 2.

EAST, E. M. I929. Self-sterility. Bibliogr. Genet., 5, 331-368.

GRABAR, P. I959. Immunoelectrophoretic analysis. Methods Biochem. Analy., 7, I -38 .

HARUTA, T. 1962. Studies on the genetics of self- and cross-incompatibility in cruciferous vegetables. (Japanese with English summary.) Research Bull. No. 2, Takii Plant Breeding and Experiment Station, Kyoto, Japan.

HAYWARD, B. J., AND AUGUSTIN, R. I957. Quantitative gel diffusion methods for assay of antigens and antibody. Int. Arch. Allergy, II, 192-206.

LEWIS, D. 1952. Serological reactions of pollen incompatibility substances. Proc. Roy. Soc. (Lond.) B, I40, I27-I 35 .

LEwIS, D. I 963 . A protein dimer hypothesis on incompatibility. Proc. IIth Int. Cong. Genet., 2, 657-663.

Linskens, H. F. 1960. Zur Frage der Entstehung der Abwehr-Korper bei der Incompatibilitat reaktion von Petunia. III. Mitteilung: Serologische Teste mit Leitgewebs-und pollen-Extrakten. Zeitschr. f. Bot., 48, I26-135.

uinskens, н. F. I 963 . Biochemistry of incompatibility. Proc. IIth Int. Cong. Genet., $2,629-636$.

MÄKINEN, Y. L. A., AND LEWIS, D. I962. Immunogenetic analysis of incompatibility (S) proteins and of cross-reacting material in a self-compatible mutant of Oenothera organensis. Genet. Res. Camb., 3, 352-363.

NASRallah, M. E. I965. Physiological and immunogenetic studies on self-incompatibility in Brassica oleracea var. capitata. Ph.D. Thesis, Cornell University, Ithaca, N.Y.

NASRALLAH, M. E., AND WALLACE, D. H. 1967 . Immunochemical detection of antigens in self-incompatibility genotypes of cabbage. Nature 213, 700-701.

PANDEY, K. K. 196o. Evolution of gametophytic and sporophytic systems of incompatibility in angiosperms. Evol., I4, 98-I I 5 .

tseng, н. 1938. Self-sterility in Antirrhinum and Petunia. 7. Genet., 36, 127-1 $3^{8 .}$ 